\title{
COMPUTATION OF AERODYNAMIC DAMPING FOR FLUTTER ANALYSIS OF A TRANSONIC FAN
}

\author{
Parthasarathy Vasanthakumar \\ Institute of Aeroelasticity \\ DLR - German Aerospace Center \\ 37073 Goettingen \\ Germany \\ EMail: Parthasarathy.vasanthakumar@dlr.de
}

\begin{abstract}
This paper describes the computational analysis of aerodynamic damping for prediction of flutter characteristics of a transonic fan stage that consists of a highly loaded rotor along with a tandem stator. Three dimensional, linearized Navier-Stokes flow solver TRACE is used to numerically analyse the flutter stability of the fan. The linear flow solver enables the modeling of a single blade passage to simulate the desired inter-blade phase angle. The unsteady aerodynamic load on a vibrating blade is obtained by solving the unsteady Navier-Stokes equations on a dynamically deforming grid and the energy exchange method is used to calculate the aerodynamic damping. The calculation of aerodynamic damping for the prediction of flutter characteristics of the fan rotor is carried out with and without considering the influence of the disk. The blade mode shapes from finite element modal analysis are obtained accordingly and the flutter calculations are carried out for three blade vibration modes at the design speed and at part speeds for all possible inter-blade phase angles. Two operating points, one on the working line and the other near stall are investigated at every rotational speed. Different aspects that affect the aerodynamic damping behaviour like part speed operation, variation in unsteady blade surface pressure fluctuation between operating points on the working line and at near stall and the corresponding variation in aerodynamic work, inter-blade phase angle etc., are described. This analysis primarily focuses on the variations in aerodynamic damping of the fan with and without the influence of the disk. In addition, influence and effect of shock wave on the aerodynamic damping is also discussed.
\end{abstract}

\section{INTRODUCTION}

The aeroelastic instability of flutter continues to occupy a predominant position in the design of turbomachinery blades. The self excited oscillation that occurs when the unsteady work performed by the fluid on the blade exceeds the energy dissipated by damping in the system is a significant problem throughout the gas turbine industry. Since damping is known to be the important parameter controlling the phenomenon of flutter, it is essential to quantify damping. In modern rotor design, blisks (integrally bladed rotors) have negligible mechanical and material damping. This leads to the fact that the unsteady aerodynamics resulting from the vibration of the blades, specifically the aerodynamic damping, plays a very significant role in the flutter analysis of these blisks.

There are two main families of techniques for numerical prediction of aerodynamic damping; nonlinear time marching and time-linearized. Despite the increase in computational power, the nonlinear time marching method in the time domain still remains a computationally expensive method to be used as a baseline design tool. In time-linearized analysis, the flow governing equations are linearized by considering the flow unsteadiness to be small compared to the mean, steady flow. The resulting perturbation equations are solved assuming that the perturbation is harmonic in the frequency domain.

The computational aeroelastic analysis for the present investigation has been carried out on a highly loaded, transonic low bypass fan designed as part of the DLR research project AeroLight. Details of the design optimization of this fan stage are given in [1] and [2]. The blade design is a result of multidisciplinary optimization, targeted on the fulfillment of multiple mechanical and aerodynamical constraints. The fan has 19 blades followed by a tandem stator. Further, a sensitivity 
study comparing the stability of different design loops has also been carried out [3]. The investigations focusing on modern turbomachinery blade row flutter and aerodynamic damping range from the sensitivity of damping calculation to changes in blade twist distribution, effect of inlet and exit conditions and time step [4] to the influence of shock and its characteristics, effects of tip clearance and vibration amplitude [5]. Unsteady shock oscillation rather than blade stall were found to be the driving mechanism for flutter instability in a transonic fan [6]. Another study showed that shock has both stabilizing and destabilizing effect with the inter-blade phase angle and location of shock having a significant impact on stability [7]. The present work focuses on computation of aerodynamic damping with and without considering the influence of the disk for prediction of flutter characteristics of the fan. The variation in aerodynamic damping between these two approaches is analyzed in detail to find the influence of blade modeshape as a factor affecting the extent of aerodynamic damping and also the influence of shock wave on damping.

\section{AERODYNAMIC DAMPING}

The aerodynamic damping parameter represents a measure of system stability, i.e. a system is stable if the aerodynamic damping parameter is greater than zero. System stability is obtained from computation of the work per cycle and its conversion to an aerodynamic damping parameter. During each cycle of vibration aerodynamic work is exchanged with the blade motion, the direction of net exchange is the criterion for aerodynamic stability. The aerodynamic work per cycle can be integrated from the unsteady surface pressure variations $(\tilde{p})$ on the blade and the local displacement velocity $(\vec{u})$ and expressed as

$$
W=\int_{0}^{T}\left[\int_{\text {Surface }}(\tilde{p} \cdot(\vec{u} \cdot \vec{n})) \cdot d \mathbf{A}\right] d t
$$

where $\vec{n}$ is the normal vector pointing outwards. A is the surface area vector and $T$ is the time period of oscillation.

A local expression of aerodynamic work per cycle can be given by

$$
W_{\text {Local }}=\int_{0}^{T}(\tilde{p} \cdot(\vec{u} \cdot \vec{n})) d t
$$

The aerodynamic work will be positive in case of destabilising flow. Positive work indicates that blade absorbs energy from the flow and the blade vibration will be amplified. For a stable motion, the work becomes negative as the flow extracts energy from the blade motion. The aerodynamic damping parameter can be expressed as the normalized form of the negative aerodynamic work. The aerodynamic damping coefficient is thus positive when the flow damps the blade vibration. Relating the aerodynamic damping to the concept of logarithmic decrement [8], damping becomes

$$
\Xi=-\frac{W}{2 \cdot K_{E}}
$$

where $K_{E}=\frac{1}{2} M \omega^{2}$ is the maximum kinetic energy over one cycle of the blade motion with $M$ being the modal mass and $\omega$ the modal frequency. A plot of aerodynamic damping for different inter-blade phase angles gives an indication of the system stability. The inter-blade phase angle is

$$
\sigma=\frac{2 \pi n}{N_{b}} ; \quad\left(n=1,2,3, \ldots, N_{b}\right)
$$

where $n$ is the number of nodal diameters and $N_{b}$ is the number of blades [9]. In flutter calculations all possible interblade phase angles may have to be checked for flutter stability to find out the least stable phase angle.

\section{NUMERICAL APPROACH}

The flow solver used in the present investigations is the three dimensional, cell centered, finite volume Reynolds averaged Navier Stokes (RANS) solver TRACE, developed at DLR Institute of Propulsion Technology, for internal flows, especially in turbomachinery [10,11]. The time-linearized solver employs the assumption of small time-harmonic perturbations and is implemented via finite differences of the nonlinear flux routines of the time accurate solver. The resulting linear system is solved in the frequency domain using a parallelized Generalized Minimal Residual (GMRES) method with a symmetric successive over relaxation (SSOR) preconditioning. The boundary conditions for solid walls are, slip condition for Euler and no-slip condition for Navier-Stokes flow. A dimensionless wall distance value of less than 25 was employed. At the inlet and exit boundaries, Giles' nonreflecting boundary conditions are employed. Turbulence closure is achieved by Wilcox k- $\omega$ two equation turbulence model. A mapping algorithm is used to map structural eigenmodes, obtained from finite element structural analysis, from the surface mesh of the finite element structural solver to the computational mesh of the finite volume flow solver. Once the displacement vectors are mapped onto the wall surfaces of the CFD mesh, the grid deformation is obtained using an elliptic mesh deformation algorithm.

The three dimensional unsteady flutter calculation requires the converged steady flow solution and modal deflections of each mode shape as input. The vibration mode shapes obtained from finite element calculations are mapped to the computational mesh to get the corresponding grid deformation for the TRACE calculations. The results of the steady simulations are used to define the rotor exit boundary conditions and initialize the flow field for the rotor unsteady 
flow simulation. The fan is designed to obtain a very high pressure ratio in a single stage configuration with transonic flows at all radial heights and a complex, highly twisted rotor blade. The computational mesh for the fan stage consists of structured multi-block mesh of nearly 1.9 million hexahedral cells. The rotor has a relative radial gap of $0.22 \%$ of the casing radius at the rotor leading edge. The rotor mesh has radially 85 mesh layers with 10 in the tip gap. Fillet radii are also considered to capture a realistic cross sectional distribution at the rotor hub.

Flutter calculations to determine the aeroelastic stability of the rotor have been carried out based on two different approaches to the finite element blade modal analysis. In the first approach, blade only geometry of the rotor without the influence of the disk has been modeled and in the second approach, the influence of the disk has also been included in the modal analysis.

Computations have been performed at design speed and several part speeds with two operating points in each speed, one at the working line and one very near stall in both approaches. In this paper, results of only $88 \%$ speed are presented and discussed as the operating point near stall at this speed showed flutter instability for the blade only modal analysis approach.

\section{RESULTS AND DISCUSSION}

The steady Mach number distribution over suction and pressure surfaces for $88 \%$ speed for the operating point near stall is shown in Figure 1a. A shock wave appears near the leading edge on the suction surface covering nearly two thirds of the blade span. The shock moves downstream on the outer regions of the blade, terminating around one third of the blade chord near the tip. On the pressure surface, the shock impinges near the blade leading edge towards the tip. Figure $1 \mathrm{~b}$ shows the Mach distribution at different spanwise locations.
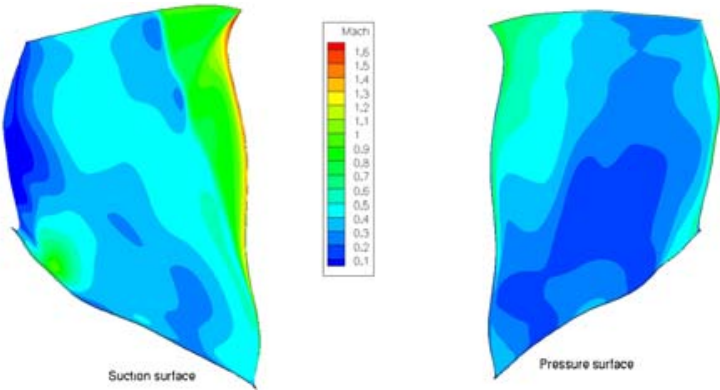

Fig. 1a Steady Mach contours

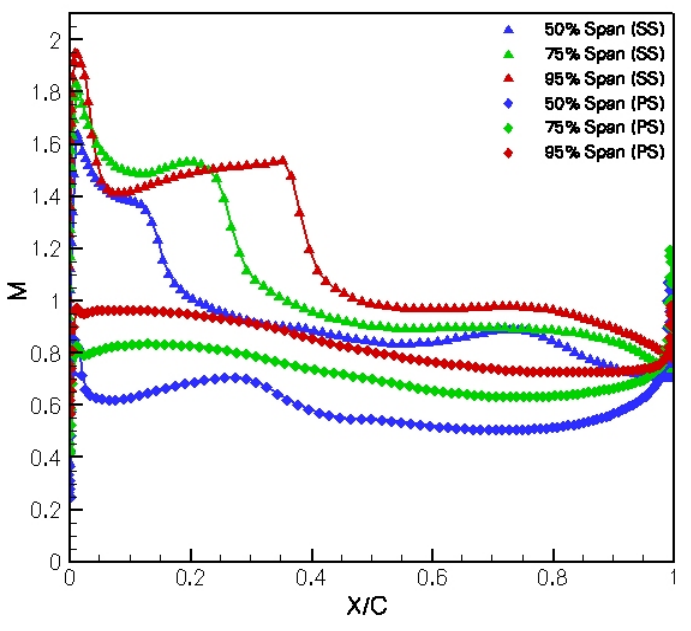

Fig. 1b Spanwise Mach number distribution

The first three blade modes from the finite element analysis were considered for the aeroelastic simulation. Figure 2 shows the blade modeshapes with and without considering the influence of the disk. The contours represent the total displacement. The first two modes, mode1 and mode2 represent the first bending and second bending respectively. The third mode, mode3 represents a combination of torsion and chordwise bending. In all the three modes, the tip region of the leading edge shows the maximum displacement. While mode1 exhibits nearly identical behaviour on the left and right sides (without and with the influence of the disk respectively), mode2 has slight variations with the left side having more displacement in the leading edge region around mid-span compared to the right side. In the case of mode3, the modeshape on the left (blade only approach, without considering the influence of the disk) shows considerable displacement on the entire outboard sections of the blade while the mode shape on the right shows the deformation restricted only to the outer section of the leading edge at the tip. For all the three modes, the approach including the influence of the disk has lower deformation in the entire leading edge region. The reduced frequencies of the three modes based on blade tip chord and inlet relative velocity are $0.2486,0.509$, and 0.6431 respectively for the blade only approach.

The plot of aerodynamic damping for 19 different inter blade phase angles (IBPA) shows that at part speed operation the rotor is stable on the working line (Fig. 3) for both approaches. The second mode has the lowest value of damping for the range of inter blade phase angles and shows least variation with phase. As the back pressure is increased and the operating point approaches near stall, there is remarkable variation in the flutter behaviour for mode2 (Fig. 4). In the blade only approach, there is a region of instability around IBPA $-113.68^{\circ}$ with negative 
aerodynamic damping. However, with the influence of the disk included, the rotor is stable for all the phase angles considered.
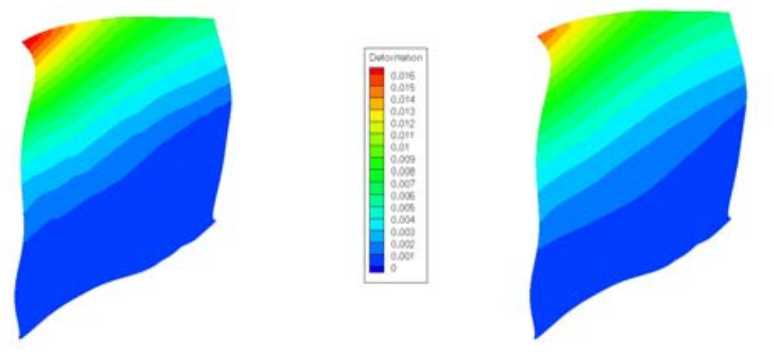

Mode1
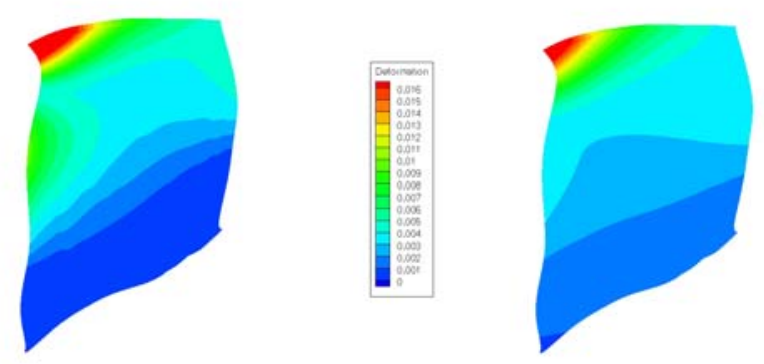

Mode2
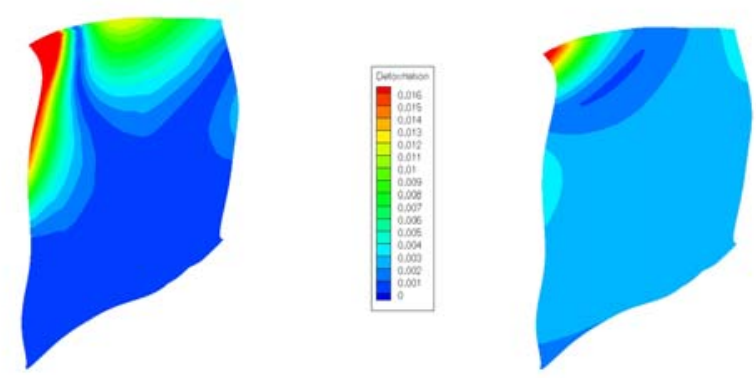

Mode3

Fig. 2 Blade modeshapes with (right) and without (left) the influence of the disk.

Figure 5 shows the distribution of local work for IBPA $113.68^{\circ}$. The large extent of positive work on the blade surface is obviously the contributing factor for the negative damping in this case. To understand this further, the local work plot has to be looked in conjunction with the plot of Mach contours shown in Figure 1. Specifically, the positive work regions on the suction surface extend from the foot of the shock wave covering the midchord region for most of the blade span and the outer region of the tip at the leading edge shows a concentration of positive work, whereas the pressure surface shows a concentration of negative work near the tip leading edge. Thus the destabilizing or stabilizing work (positive or negative) is associated with the shock wave location. For the backward traveling wave in this case (negative IBPA), the vibratory pattern moves opposite to the direction of rotation. Therefore, a stabilizing effect on the pressure surface and a destabilizing effect on the suction surface around the shock location enhance the net destabilizing effect. It should also be noted that the region of the leading edge where the local work shows maximum magnitude corresponds to the same location where the modal displacement is also the maximum. The adjoining IBPA of $-94.74^{\circ}$ (Fig. 5 (b)) exhibits a very similar work pattern with slightly less intensity.

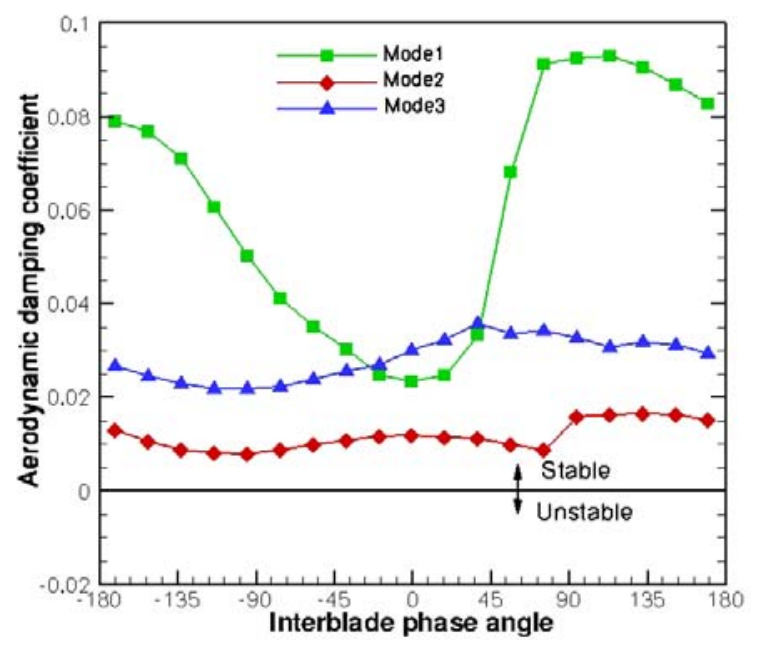

(a) Blade only approach

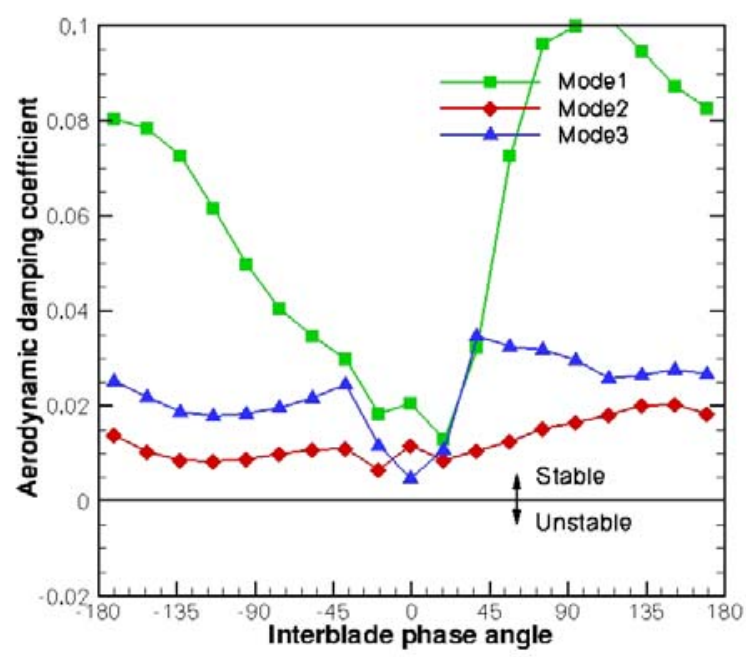

(b) With influence of the disk

Fig. 3 Aerodynamic damping for working line operation 


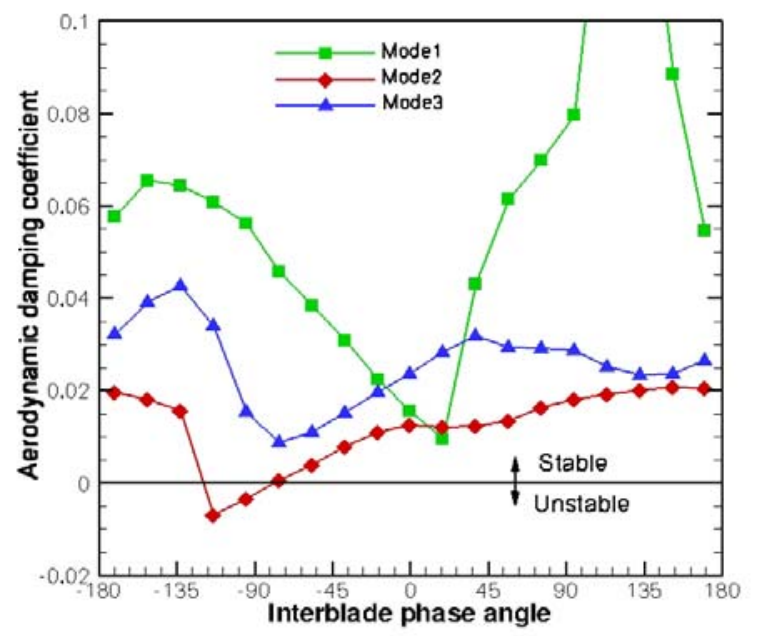

(a) Blade only approach

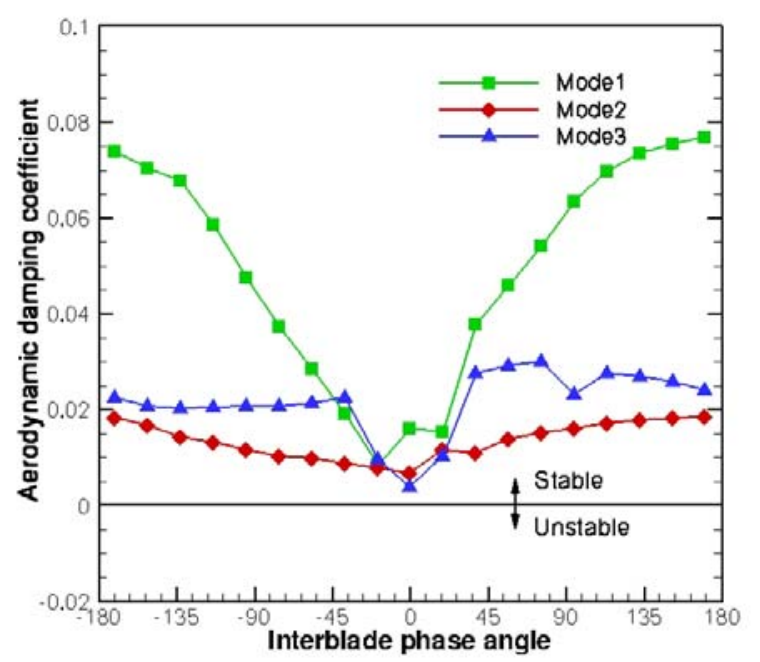

(b) With influence of the disk

\section{Fig. 4 Aerodynamic damping for near stall operation}

However, for other IBPAs, the same shock pattern has not produced any destabilizing effect in the blade only approach. This is due to the fact that the work done on the blade depends not only on the shock impulse but also on the phase relationship between the pressure fluctuation and the blade motion. As the IBPA is changed, it alters the phase between the unsteady pressure fluctuation associated with the shock motion and the blade motion. Therefore, in addition to the shock location and strength, the phase relation between the blade motion and shock motion is also needed to establish why in some cases the work is destabilizing and why in other cases it is not. On the other hand, when the influence of the disk is also included, Fig. 4 shows that the aerodynamic damping is positive for all IBPAs of mode2. A look at the local work distribution for IBPA $-113.68^{\circ}$ of mode2 in this case, Fig. 6, shows that on the suction surface, though the positive work region covers almost the entire leading edge, it is of the same order as in other regions of positive work. Also, the pressure surface leading edge shows positive work distribution and overall the positive work region is more concentrated on the lower half of the blade span on both surfaces contributing less to the destabilizing effect. For IBPA $-94.74^{\circ}$, almost the entire leading edge is free of positive work on both surfaces. This difference in the damping behaviour between the two approaches for mode2 shows that even a small variation in modal displacement at the leading edge region greatly influences the stability of the blade.

Figure 7a gives the spanwise distribution of damping for IBPA $-113.68^{\circ}$. It is clear that for the blade only approach there is large variation in damping in the upper half of the blade between $60 \%$ and $95 \%$ of the span compared to the case when influence of the disk is included. Figure $7 \mathrm{~b}$ shows the chordwise distribution of damping at 95\% span for IBPA $113.68^{\circ}$. In the blade only approach, the leading edge region has a larger influence compared to other regions and there is considerable variation in damping on both surfaces in this region. When the influence of the disk is also considered, the variations in the leading edge region are greatly reduced. Figure 7a also shows that the blade only approach has the lowest damping around $70 \%$ of the span. The chordwise distribution of damping at $70 \%$ of span for IBPA $-113.68^{\circ}$ is given in Figure 7c. Again, the leading edge region seems to have a greater influence on the damping behaviour of the blade only approach. However, the stability behaviour of the suction and pressure surfaces varies between $95 \%$ and $70 \%$ span. This raises a question on the role of shock in influencing instability. From figures $1 \mathrm{a}$ and $1 \mathrm{~b}$, it is seen that the foot of the shock extends from around $15 \%$ of the blade chord at midspan to around $35 \%$ of the chord at the tip. The variation in the stability behaviour between $95 \%$ and $70 \%$ span suggests that the shock impulse at $95 \%$ span is not wide enough to cover the entire leading edge region. At the same time, the transonic flow condition on the pressure surface at $95 \%$ span in the leading edge region seems to cause the unsteady blade surface pressure at this location a source of instability. At span locations below $85 \%$, the reach of the shock oscillation appears to cover the leading edge region well enough to cause instability on the suction surface.

In order to get further insight on this, understanding the variations in the damping behavior for mode3 is also warranted. IBPA $0^{\circ}$ of mode 3 provides the least damping when the influence of the disk is included (Fig. 4). Comparing the local work distribution for IBPA $0^{\circ}$ in this case with the blade only approach in Figure 8, we find that the leading edge region on the suction surface in the latter case has a strong stabilizing 
effect (negative work) compared to the former. Also, with the blade only approach, even though the extent of positive work region is larger and coincides with the location of the foot of the shock on the suction surface compared with the other approach, the stabilizing effect is better. As seen from Figure 2 , the blade only approach has displacement for nearly two thirds of the blade leading edge, while the other has displacement for less than one third of the leading edge. In the absence of any phase difference, this effect is stronger and hence the least damping for IBPA $0^{\circ}$ when the influence of the disk is included. This clearly indicates that the variation in leading edge deformation between the two approaches strongly influences the damping behavior. Since other IBPAs of mode3 do not show much variation in damping between the two approaches, one other IBPA than these ones should be checked to find out if phase angle variation plays any role. Figure 9 shows the local work distribution for IBPA 56.84 for both approaches. Here again, the leading edge region on the suction
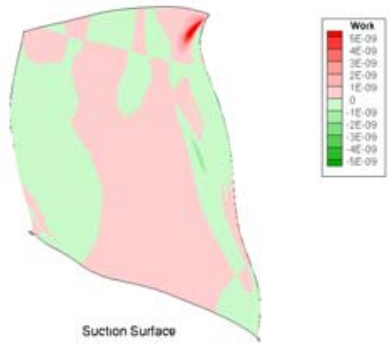

(a) IBPA $-113.68^{\circ}$

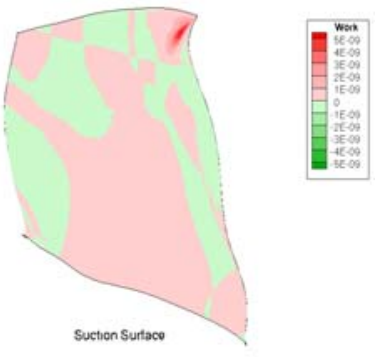

(b) IBPA $-94.74^{\circ}$

Fig.5 Local work distribution (Blade only approach; Mode2)
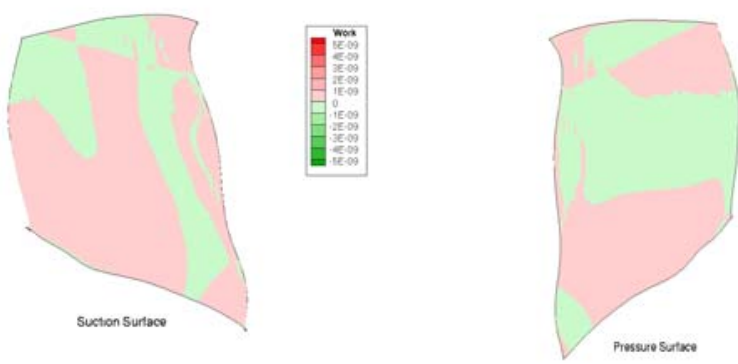

(a) IBPA -113.68
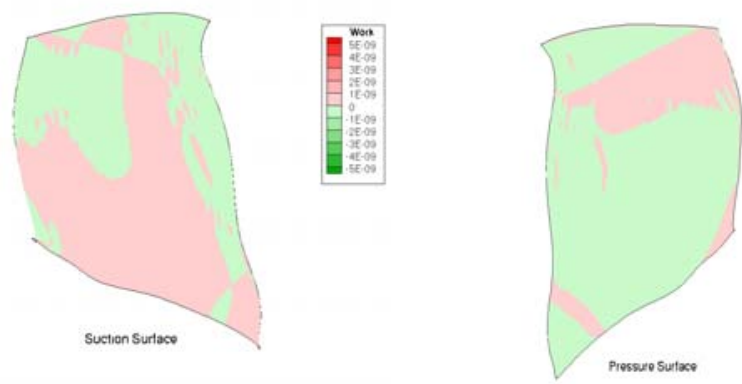

(b) IBPA -94.74 ${ }^{\circ}$

Fig. 6 Local work distribution (With influence of disk; Mode2)

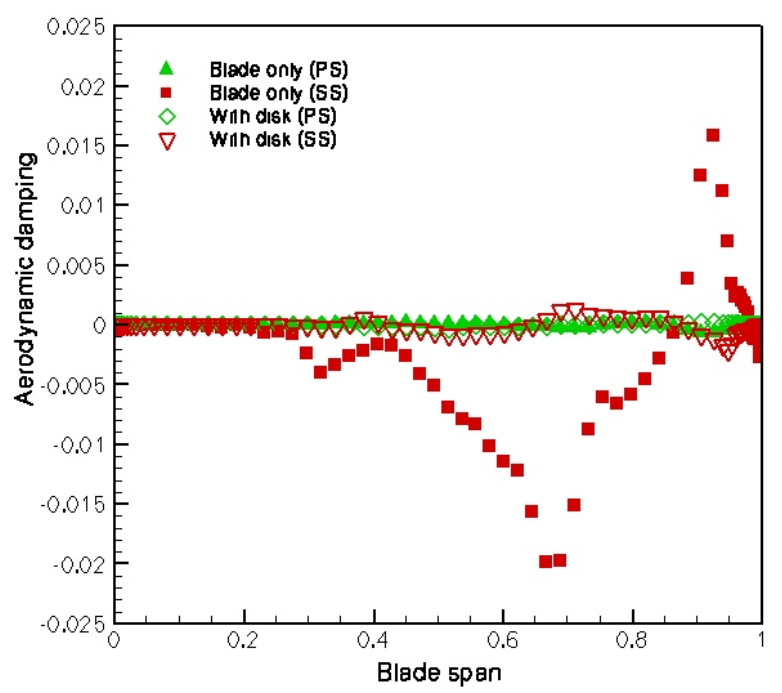

Fig 7a Spanwise distribution of damping for IBPA $-113.68^{\circ}$ 


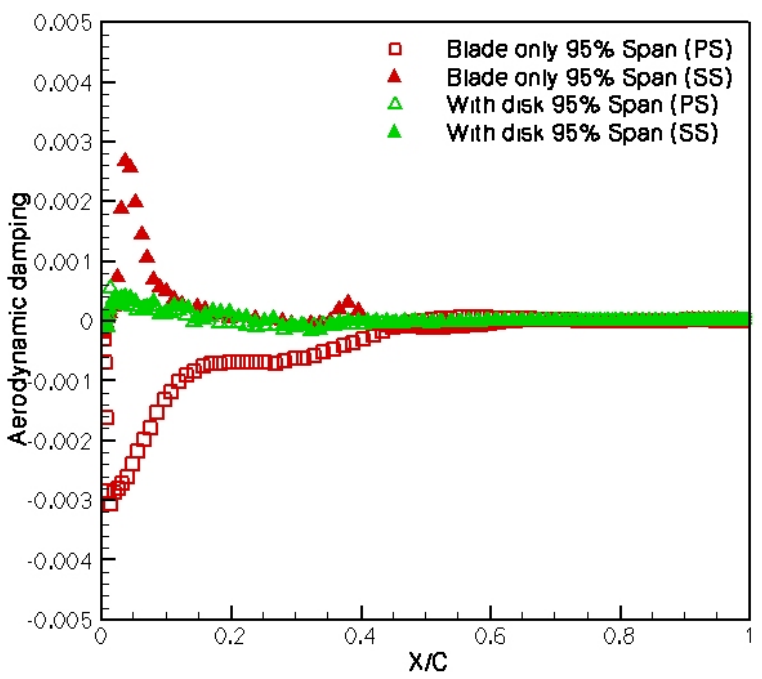

Fig $7 b$ Chordwise distribution of damping at $95 \%$ span $\left(\right.$ IBPA $\left.=-\mathbf{- 1 1 3 . 6 8}^{\circ}\right)$

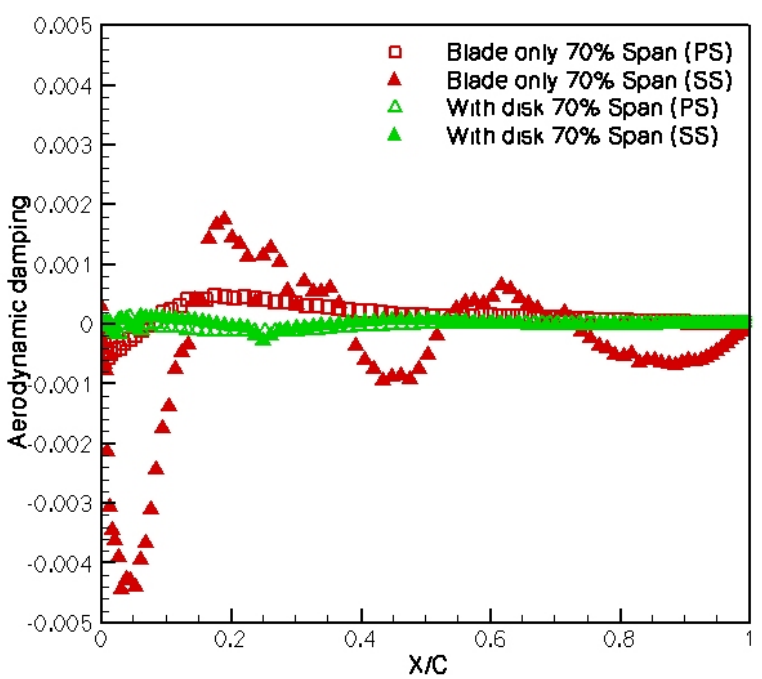

Fig 7c Chordwise distribution of damping at $70 \%$ span $\left(\right.$ IBPA $\left.=-113.68^{\circ}\right)$

surface for the blade only approach has a stronger negative work region compared to the other approach. However, the phase difference appears to reduce the damping in the blade only approach and increase it in the other approach. The exact nature of how this takes effect is, however, unclear.

Based on the above observations, factors affecting the aerodynamic damping and the stability of the blade are, firstly, the blade modeshape and secondly, the influence of the shock coupled with the inter blade phase angle. The present work indicates that even a slight variation in modal deformation can
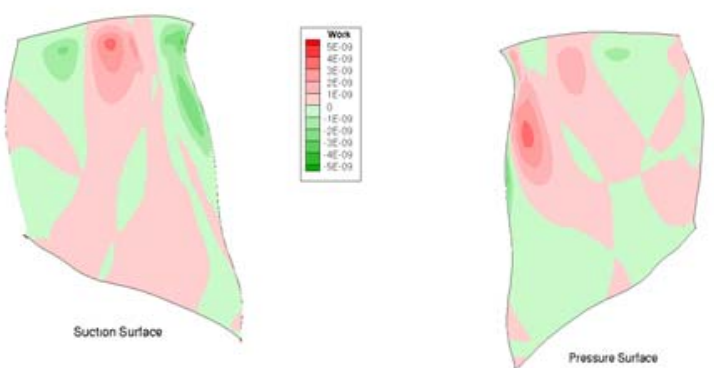

(a) Blade only approach
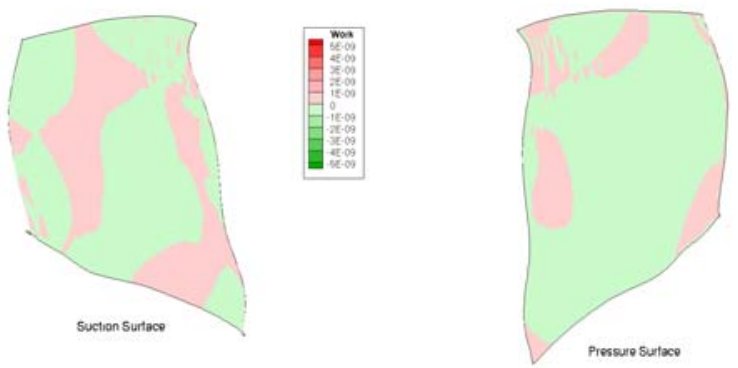

(b) With influence of the disk

Fig. 8 Local work distribution for IBPA $0^{\circ}$ (Mode3)
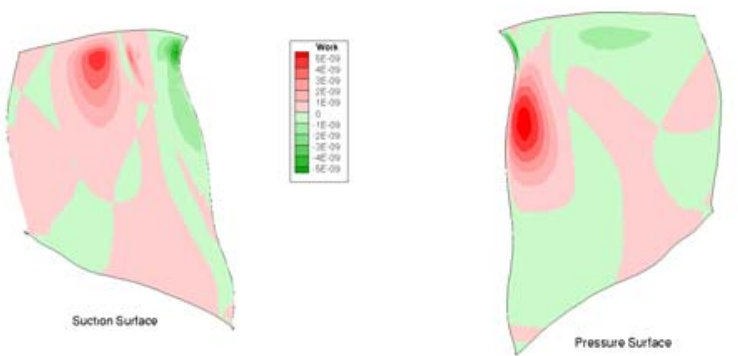

(a) Blade only approach
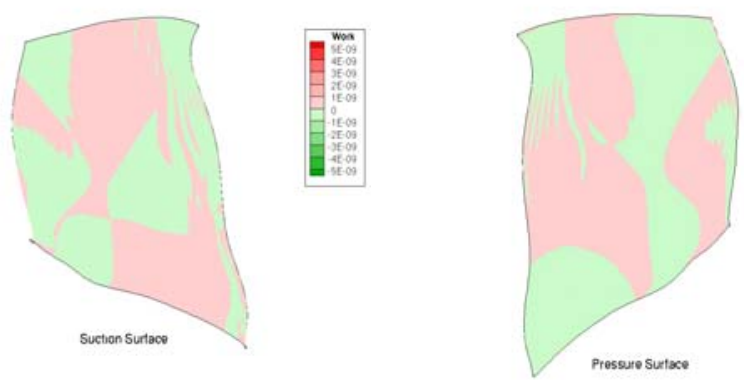

(b) With influence of disk

Fig. 9 Local work distribution for IBPA 56.84 (Mode3) 
lead to large changes in the aerodynamic damping behavior. Modal analysis with the blade only approach is too conservative. Therefore, modal deformation input based on the inclusion of the influence of the disk is the appropriate approach. That such large variation could be seen in a blisk is indicative of how unsteady aerodynamics from the blade motion and consequently the aerodynamic damping is the major factor for blade stability. This reinforces the observation that blade mode shape is an important in determining blade stability [12]. In addition, the location of the shock also plays a role in contributing to the stabilizing or destabilizing effect depending on the inter blade phase angle. This explains how in certain IBPAs the work contribution is negative and in some other cases it is positive at the location of the shock. This also concurs with the observation in [7]. However, further detailed analysis is required to establish the concrete relationship between shock motion and inter blade phase angle. A comparison of the linearized Euler and Navier-Stokes solution for this rotor for near stall operating points [13] also suggests that shock motion near tip region is a contributing factor for instability. When the influence of the disk is included, the blisk structure exhibits cyclic symmetry with complex mode shapes. The large variation in damping between the blade only approach and with the influence of the disk shows that cyclic symmetric effects play a significant role in the damping behavior as observed in [14].

\section{CONCLUSIONS}

The aeroelastic stability of a highly loaded transonic fan has been investigated by computing the aerodynamic damping of the fan using the linearized Navier-Stokes solver TRACE. The unsteady computations were based on two different approaches to the blade modal analysis, one with the blade only approach and the other including the influence of the disk. The first three blade modes were considered for the investigations. Aerodynamic damping for all possible inter blade phase angles have been computed and results of the calculations for $88 \%$ speed have been presented and discussed. The operating point near stall showed instability for the blade only approach as the aerodynamic damping was negative for two IBPAs. Analysis of the local work distribution for different IBPAs has indicated that the aerodynamic damping of the blade is largely influenced by the blade mode shape and the shock location. Even a slight variation in the modeshape causes changes in the damping and subsequently the stability.

Thus the blade mode shape is a very important factor in determining stability. The shock location and movement and its relation to the inter blade phase angle also contributes significantly to the variation in the aerodynamic damping. The exact nature of this relation needs further analysis.

\section{ACKNOWLEDGMENT}

The support of this work by the German Federal Ministry of Defence is gratefully acknowledged. The author would like to thank C. Frey, C. Voigt, G. Ashcroft and U. Siller from the DLR Institute of Propulsion Technology for their help and support for TRACE and for providing the fan input data. Thanks are also due to P. Ebel from DLR Institute of Structures and Design for providing the modal data from finite element analysis. The author would also like to thank his colleagues J. Belz and M. May for the stimulating discussions.

\section{REFERENCES}

[1] Siller, U., Voss, C., and Nicke, E., 2009, “Automated Multi-disciplinary Optimization of a Transonic Axial Compressor,” AIAA Paper 2009-863.

[2] Siller, U., and Aulich, M., 2010, "Multidisciplinary 3D Optimization of a Fan Stage Performance Map with Consideration of the Static and Dynamic Rotor Mechanics,"ASME Paper GT2010-22792

[3] May, M., 2010, "Sensitivity Analysis With Respect to Flutter Free Design of Compressor Blades," ASME Paper GT2010-23557

[4] Srivatsava, R., Bakhle, M.A., and Keith Jr., T.G., 2003, "Numerical Simulation of Aerodynamic Damping for Flutter Analysis of Turbomachinery Blade Rows," AIAA Journal of Propulsion and Power, Vol. 19, No.2, pp. 260-267

[5] Sanders, A.J., Hassen, K.K., and Rabe, D.C., 2004, "Experimental and Numerical Study of Stall Flutter in a Transonic Low Aspect Ratio Fan Blisk,” Trans ASME Journal of Turbomachinery, Vol. 126, pp. 166-174.

[6] Isomura, K., and Giles, M.B., 1998, “A Numerical Study of Flutter in a Transonic Fan," Trans ASME Journal of Turbomachinery, Vol. 120, pp. 500-507.

[7] Srivatsava, R., and Keith Jr., T.G., 2005, "Influence of Shock Wave on Turbomachinery Blade Row Flutter," AIAA Journal of Propulsion and Power, Vol. 21, No.1, pp. 167-174

[8] Carta, F.O., 1967, "Coupled Blade-Disk-Shroud Flutter Instabilities in Turbojet Engine Rotors,” Trans ASME Journal of Engineering for Power, Vol. 89, No.3, pp. 419- 426.

[9] Lane, F., 1956, "System Mode Shapes in the Flutter of Compressor Blade Rows," Journal of the Aeronautical Sciences, Vol. 23, No.1, pp. 54-66.

[10] Petrie-Repar, P., 2006, "Development of an Efficient and Robust Linearized Flow Solver,“ in Unsteady Aerodynamics, Aeroacoustics and Aeroelasticity of Turbomachines, K.C. Hall et al. (eds.), pp. 437-448, Springer Netherlands.

[11] Kerksen, H-P., Frey, C., Voigt, C., and Ashcroft, G., 2010, "Time Linearized and Time Accurate 3D RANS Methods for Aeroelastic Analysis in Turbomachinery," ASME Paper GT2010-22940

[12] Panovsky, J., and Kielb, R. E., 1998, “A Design Method to Prevent Low Pressure Turbine Blade Flutter,” ASME Paper No. 98-GT-575.

[13] May, M., and Grüber, B., 2011,” Reliability of Flutter Predictions with Time-Linearized Flow solvers for Operating Points Near the Surge Line," Paper submitted for approval to the $9^{\text {th }}$ European Turbomachinery Conference, Istanbul, Turkey, March, 2011 
[14] Kielb, R., Barter, J., Chernysheva, O., and Fransson, T., 2006, "Flutter Design of Low Pressure Turbine Blades with Cyclic Symmetric Modes," in Unsteady Aerodynamics, Aeroacoustics and Aeroelasticity of Turbomachines, K.C. Hall et al. (eds.), pp. 41-52, Springer Netherlands. 